Revista de la Escuela de Ciencias de la Educación, año 15, nRo. 14, vol. 2, Julio a Diciembre de 2019. PÁGinas 51-60. ISSN 18516297 (DESDE DICIEMBRE DE 2006 A DICIEMBRE DE 2017). ISSN 2362-3349 (EN LÍNEA). DOCENCIA, TRABAJO Y DISPUTAS COTIDIANAS EN ESCUELAS SECUNDARIAS DE LAS SOCIEDADES DEL GERENCIAMIENTO. EdUARDO LANGER. GabRIELA ORLANDO.

\title{
DOCENCIA, TRABAJO Y DISPUTAS COTIDIANAS EN ESCUELAS SECUNDARIAS DE LAS SOCIEDADES DEL GERENCIAMIENTO
}

\author{
Eduardo Langer* \\ Universidad Nacional de San Martín, Argentina \\ langereduardo@gmail.com \\ Gabriela Orlando* \\ Universidad Nacional del Luján, Argentina \\ orlandogabriela@gmail.com
}

Recibido: 17/10/2018 Aceptado: 22/11/2018

\begin{abstract}
Resumen
En este artículo se caracteriza la docencia y el trabajo cotidiano en las escuelas atendiendo a sus prácticas discursivas en torno a las nuevas formas de regulación de la escolaridad, así como a las tensiones, contradicciones y disputas que van desplegando en la cotidianeidad educativa. Se sostendrá como hipótesis que, en condiciones socio-educativas de fragmentación y desigualdad, las estrategias de gobierno que alientan el emprendedurismo suponen una sobrecarga de los docentes que son señalados directamente en el sostenimiento de las escuelas. A la vez, en ese proceso "dialogan" en y con esas políticas, en y con las condiciones que atraviesan a las escuelas y, en vez de la negación, activan respuestas y disputan sentidos a través de la inventiva y la creación para poder seguir enseñando. El estudio se desarrolla a través de una propuesta metodológica cualitativa y múltiple; esto es, la realización de entrevistas en profundidad a docentes del nivel secundario durante 2016 y 2017, así como observaciones en un taller audiovisual que se viene desplegando desde el 2008 a la actualidad en una escuela secundaria del Partido de Gral. San Martín y, por último, a través de resultados de una acción de extensión que involucra a todas las escuelas secundarias del mismo partido.
\end{abstract}

Palabras Clave: Docencia - Trabajo - Regulación - Disputas - Escuelas secundarias.

Abstract

In this article, we discuss the teaching activities and the daily work of teachers in the schools. We pay attention to their discursive practices around the new forms of schooling regulation, with a focus on the tensions, contradictions and disputes of their daily activities. The hypothesis is that, under socio-educational conditions of fragmentation and inequality, the government strategies that encourage the entrepreneurship of teachers represent an overload for

\footnotetext{
* Post Doctor (Universidad Nacional de San Martín), Doctor en Educación (Universidad de Buenos Aires), Magister en Ciencias Sociales con mención en Educación (FLACSO/Argentina) y Licenciado en Ciencias de la Educación (Universidad de Buenos Aires). Es Investigador Adjunto del Consejo Nacional de Investigaciones Científicas y Técnicas (CONICET), es miembro del CEDESI/EHU/UNSAM (Centro de Estudios en Desigualdades, Sujetos e Instituciones/Escuela de Humanidades/ Universidad Nacional de San Martín), es Director de las carreras de Educación en la Universidad Nacional de San Martín y se desempeña como Profesor de Sociología de la Educación por la Universidad Nacional de General San Martín y la Universidad Nacional de la Patagonia Austral.

* Magister en Política y Gestión de la Educación (Universidad Nacional de Luján-UNLU), Licenciada y Profesora en Ciencias de la Educación (UNLU) y Profesora de Educación Preescolar (ISFD № 110). Es miembro del CEDESI/EHU/UNSAM (Centro de Estudios en Desigualdades, Sujetos e Instituciones/Escuela de Humanidades/ Universidad Nacional de San Martín). Realiza la asistencia de Dirección de las Carreras de Educación en la UNSAM y se desempeña como Jefa de Trabajos Prácticos en Investigación Educativa (UNSAM) y en el Área de Metodología de investigación (UNLU) y como profesora en diversas asignaturas en el ISFD № 110 de Moreno, Provincia de Buenos Aires. Sus temas de investigación abrevan en los estudios de gubernamentalidad y educación y refieren a las políticas de escolarización y el trabajo cotidiano de los docentes, enfocando, especialmente, en las condiciones institucionales de las escuelas públicas en contextos de pobreza urbana.
} 
Revista de la Escuela de Ciencias de la Educación, año 15, nRo. 14, vol. 2, Julio a Diciembre de 2019. PÁGinas 51-60. ISSN 18516297 (DESDE DICIEMBRE DE 2006 A DICIEMBRE DE 2017). ISSN 2362-3349 (EN LÍNEA). DOCENCIA, TRABAJO Y DISPUTAS COTIDIANAS EN ESCUELAS SECUNDARIAS DE LAS SOCIEDADES DEL GERENCIAMIENTO. EDUARDO LANGER. GabRIELA ORLANDO.

their work when trying to keep schools sustainable. However, teachers respond to these policies and to the conditions of the schools; and instead of rejecting them; teachers dispute the sense of these policies through their inventiveness and through the re-creation of their capabilities in order to continue their teaching. This study is developed through a qualitative and multiple methodological methods approach. We developed in-depth interviews with secondary level teachers in 2016 and 2017, as well as observations in an audiovisual workshop which has been unfolding since 2008 in a secondary school of the General San Martín District. In addition, other results and data came from a community project involving all high schools of the same District.

Key Words: Teaching - Job - Regulation - Disputes - High schools.

\section{Introducción}

Como agentes estatales, los docentes trabajan, son formados, producidos con y en nuevas lógicas de gobierno basadas, entre otros aspectos, en el traslado de responsabilidades a la comunidad y los individuos, en un contexto cada vez más globalizado y atravesado por las lógicas de las competencias. De ahí que, en la actualidad, se produce toda una batería de regulaciones dirigidas a las actividades escolares de los docentes, a su trabajo. Éstas quedan expresadas en una multiplicidad de discursos y prácticas de gobierno (Miller y Rose, 1990; Foucault, 2009). Así, por ejemplo, en la nueva secundaria que oficialmente se viene diseñando en la Argentina del siglo XXI, estos discursos y prácticas pautan exhaustivamente cómo organizar tiempos y espacios con el argumento de hacerlos más aprovechables que antes. En este artículo se caracterizará la docencia y el trabajo cotidiano en las escuelas atendiendo a sus prácticas discursivas en torno a esas nuevas formas de regulación de la escolaridad, así como a las tensiones, contradicciones y disputas que van dando los sujetos en la cotidianeidad educativa.

Se busca aquí la caracterización de la vida social y educativa a través de las relaciones de fuerza que, más que a disciplinar a la población, se orientan a producir sujetos autónomos, que se construyan a -y se hagan responsables de- sí mismos, capaces de asumir y gestionar riesgos (Foucault, 2006; O' Malley, 2014), de crear e incorporarse a redes o de ser emprendedores de sus propias vidas (Rose, 2007; Grinberg, 2008). Nuestro estudio se sitúa en el pasaje de las sociedades disciplinarias (Foucault, 2002) a las sociedades de gerenciamiento (Grinberg, 2008); un pasaje que supone transformaciones que involucran, necesariamente, a las instituciones -no sólo las escuelas, ni sólo las secundarias en que se focaliza en este artículo- y a la producción de subjetividades. En el marco del gerenciamiento, el Estado está presente de una manera diferente a la de épocas anteriores. No para vigilar a todos y castigar a los que se desvían de la norma (Foucault, 2006) sino que mantiene el orden a través del control, más que sobre los individuos, sobre el medio. Se trata de un Estado que deja hacer a los sujetos para actuar apenas antes de que todo estalle (Grinberg, 2009) o aún luego de que todo estalla (1).

En este artículo, se sostendrá como hipótesis que, en condiciones socio-educativas de fragmentación y desigualdad, las estrategias de gobierno que alientan el emprendedurismo, suponen una sobrecarga y súper-responsabilización (Murúa, Orlando y Paredes, 2016) de los sujetos que son señalados directamente en el sostenimiento de las escuelas: los docentes. A la vez, esos sujetos en ese proceso "dialogan" en y con esas políticas, en y con las condiciones que atraviesan las escuelas y, en vez de la negación, activan respuestas y disputan sentidos a través de la inventiva y la creación para poder seguir enseñando.

Atendiendo a lo anterior, en este artículo se caracterizan las formas en que esos procesos de responsabilización y sobrecarga se presentan en el trabajo cotidiano de los docentes y se despliegan en prácticas que, en lugar de desplazarlos de sus responsabilidades, los llevan a asumirlas de manera más intensa. Asimismo, se asume la descripción de la cotidianeidad de ese trabajo en términos de una positividad productiva que, aun en la sobrecarga, pone en marcha la inventiva y la creación, a través de un poder productivo y móvil que "afecta de maneras novedosas las posibilidades de acción de los otros" (Langer, 2016, p.266) para pensar, proponer y hacer algo diferente en el proceso educativo. Antes de ello, a continuación, se describen en unas breves líneas el proceso de la investigación.

\section{Breves notas de la metodología utilizada}

Las investigaciones que enmarcan el presente artículo se desarrollan, desde hace una década, en escuelas secundarias públicas emplazadas en contextos de extrema pobreza urbana y degradación ambiental en el Partido de Gral. San Martín, provincia de Buenos Aires. Desde una perspectiva cualitativa, la indagación se orienta a la comprensión de la vida cotidiana de los sujetos en las instituciones. Específicamente, aquí se presenta una descripción del trabajo cotidiano de los docentes y las tensiones, contradicciones y disputas que ellos dan en el cotidiano escolar.

Aquí, los sujetos no son concebidos como quienes actúan por sí mismos e individualmente (Deleuze, 2015). Esto es, la inscripción en posiciones o lugares que cada uno ocupa en una producción no es individual sino colectiva. Desde esta perspectiva, se habla, se percibe el Estado, se vive, se hace docencia en las escuelas secundarias. Aun cuando sea una persona quien habla, lo que hace-dice 
Revista de la Escuela de Ciencias de la Educación, año 15, nRo. 14, vol. 2, Julio a Diciembre de 2019. PÁGINAS 51-60. ISSN 18516297 (DESDE DICIEMBRE DE 2006 A DICIEMBRE DE 2017). ISSN 2362-3349 (EN LÍNEA). DOCENCIA, TRABAJO Y DISPUTAS COTIDIANAS EN ESCUELAS SECUNDARIAS DE LAS SOCIEDADES DEL GERENCIAMIENTO. EdUARDO LANGER. GabRIELA ORLANDO.

ocupa un lugar en los haceres-decires colectivos, realiza una efectuación del se dentro de una familia de enunciados (Deleuze, 2015) y de prácticas. Así, la perspectiva expresada por un profesor ocupa y muestra un posicionamiento que no es individual. Esa inscripción en el se no pierde de vista el detalle, ni lo pretende. Muy por el contrario, se fija y busca lo minúsculo e infinitesimal que aparece, por ejemplo, en las expresiones de los entrevistados. Ello en tanto que es allí, en esas prácticas de hormiga (De Certeau, 2010) que lo nuevo, lo productivo tiene su espacio de aparición, como respuesta, como diálogo o como contrapropuesta a las regulaciones estatales. Ese análisis detallado y detallista, requiere prestar atención a las cotidianeidades, porque es allí donde el camino de las hormigas puede seguirse y estudiarse, donde lo habitual puede ser desnaturalizado para comprender las prácticas cotidianas de los sujetos. A la vez como campo e instrumento de lucha, la vida cotidiana se vincula con las producciones humanas y abre posibilidades de estudiar esos múltiples haceres que involucra el trabajo cotidiano de los docentes.

Así, aquí se propone abordar las prácticas discursivas sobre el hacer docencia y sobre el trabajo cotidiano en las escuelas, atendiendo a esas nuevas formas de regulación y a las disputas que activan los sujetos en y con ellas. Ello en el marco de una investigación (2) que se sostiene en una propuesta metodológica cualitativa y múltiple; esto es, la realización de entrevistas en profundidad a docentes del nivel secundario durante 2015, 2016 y 2017, así como observaciones en un taller audiovisual (3) que se viene realizando desde el 2008 a la actualidad en una escuela secundaria del Partido de Gral. San Martín y, por último, a través de resultados de una acción de extensión que involucra a todas las escuelas secundarias del mismo partido (4).

Se caracterizan las prácticas discursivas de docentes que trabajan en escuelas secundarias emplazadas en contextos de pobreza urbana. Es decir, los sujetos a quienes refiere esta investigación no son cualquier docente, sino aquellos que tienen presencia efectiva y simbólica frente y junto a sus estudiantes; son docentes valorados por pares y por la comunidad educativa. Docentes que se constituyen en referentes de sus alumnos: entre otras cosas, porque les enseñan contenidos propios de su materia que los entusiasman, preparan los materiales antes de dar clase, proponen salidas didácticas, participan con sus estudiantes de actividades de articulación entre la escuela y la universidad y, además, los escuchan y aconsejan respecto de problemáticas personales, acompañan situaciones de maternidad o paternidad adolescente, realizan arreglos edilicios en las escuelas. Se los puede ver conversando con estudiantes en los recreos, transmitiendo y distribuyendo saberes aun fuera de las aulas o realizando actividades en la escuela los días feriados. En situaciones desarrolladas en la universidad, se puede observar que los jóvenes los requieren, los esperan para exponer frente a ellos sus producciones: su presencia es constante y resulta significativa. A su vez, sus prácticas cotidianas, más que en configuraciones binarias o dualistas, se inscriben en composiciones fractales (Lazzarato, 2010). Justamente, es a esta fractalidad que presenta el trabajo docente en las escuelas, que se refiere a continuación.

\section{La fractalidad del trabajo docente}

Concebir al trabajo de los docentes a partir de la noción de fractalidad requiere que se lo comprenda en la confluencia de una serie de problematizaciones. Entre ellas, se destacan: el abordaje de las prácticas en la filigrana (Foucault, 2007; Giard, 2010) de la cotidianeidad; la necesidad de puesta en situación de esas prácticas, lo que algunos denominan como prácticas situadas (Ezpeleta, 1991) y otros como experiencia sitiada (Dubet, 2006); la noción de prácticas que, por reguladas, no niegan el hacer creativo; el trabajo de los docentes que, como conducción de conductas, involucra el vínculo con otros y consigo mismos; la idea de trabajo como práctica de resistencia y lucha (Langer, 2016, 2017; Imen, 2008).

Desde esta perspectiva, referir al trabajo en la vida cotidiana de las escuelas supone concebirlo como hacer, en sus aspectos minúsculos, infinitesimales (De Certeau, 2010) por lo múltiple, innumerable e invisible -salvo que se mire en detalle- de sus prácticas. Una labor que, en sordina, se aprecia en la multiplicidad de lo cotidiano y que, desde allí, construye apoyos, afectos y efectos que resignifican el orden dominante desde abajo y desde adentro, lo metamorfosean, le incorporan reglas propias y, en la medida de las nuevas posibilidades que abren, lo ponen en consonancia con intereses, gustos, criterios, deseos, perspectivas, problemáticas de los propios sujetos, que son autores y actores (Giard, 2010) de la vida escolar. Ese trabajo involucra aquello que hacen los docentes en y con unas determinadas condiciones, mediatas -las regulaciones, normas, cultura institucional, pautas de desempeño- e inmediatas -el espacio escolar, con sus útiles, sus muebles; los tiempos de que se dispone y las formaciones grupales- (Rockwell, 2018).

Importan, aquí, las expresiones de los sujetos respecto de ese trabajo, sumamente creativo y cómo se imbrica con unas condiciones escolares en el devenir diario de las prácticas, en sociedades que, justamente, por asumir una lógica gerencial, llaman a los sujetos y las comunidades a actuar, a empoderarse (Grinberg, 2008). Si, como se piensa aquí, el trabajo de los docentes trata tanto de las prácticas de enseñanza cotidianas como de las prácticas regulatorias desde las que se procura configurarlo, será posible describir líneas descendentes y líneas ascendentes en el ejercicio del poder (Foucault, 2007a). Es a la comunicación, continuidad y entrecruzamiento de esas líneas que se refiere en este artículo, en tanto expresan la multiplicidad, la creatividad, la productividad y las luchas presentes en las prácticas cotidianas en las escuelas. 
Revista de la Escuela de Ciencias de la Educación, año 15, nRo. 14, vol. 2, Julio a Diciembre de 2019. PÁginas 51-60. ISSN 18516297 (DESDE DICIEMBRE DE 2006 A DICIEMBRE DE 2017). ISSN 2362-3349 (EN LÍNEA). DOCENCIA, TRABAJO Y DISPUTAS COTIDIANAS EN ESCUELAS SECUNDARIAS DE LAS SOCIEDADES DEL GERENCIAMIENTO. EDUARDO LANGER. GabRIELA ORLANDO.

Las líneas descendentes involucran, predominantemente, las prácticas discursivas relativas a los procesos de escolarización y al trabajo de los docentes como se expresan en una serie de documentos oficiales y en lo que afectan a la cotidianeidad escolar. Pero ese discurso oficial no se recibe en las escuelas sin más, sino que sufre múltiples apropiaciones, lecturas, relecturas, transformaciones y resignificaciones (Beltrán Llavador, 2006). Desde ahí, el trabajo de los docentes es una práctica afectada por una multiplicidad de regulaciones (Feldfeber, 2007; Langer, 2017; Lazzarato, 2010), que no provienen sólo y exclusivamente "desde arriba", sino también "desde abajo", o "desde adentro" del propio cuerpo docente. Especialmente en tiempos de gerenciamiento, esas regulaciones entrecruzan diversos procesos y dispositivos de producción de categorías consideradas científicas, de enunciación de lo legal, de gestión, desplegados tanto desde las agencias oficiales como desde los propios sujetos. Como ya se expresó, se trata de técnicas, saberes, procedimientos y formas de enunciar que, no sólo o más que en la regulación externa, se sostienen en la auto-regulación, la autoconducción.

Así, si bien aquí se reconoce al trabajo, al empleo y al desempleo en general como actividades no naturales, sino reguladas "por las normas, las costumbres, los saberes, las prescripciones religiosas, mediáticas y culturales, por una multiplicidad de dispositivos [...] diferentes técnicas y procedimientos, por diferentes saberes y modos de enunciación" (Lazzarato, 2010, pp. 17-18), se pone el énfasis en el trabajo cotidiano como hacer; un hacer que también puede implicar no hacer. Hacer y no hacer constituyen, ambos, modos de resistir y rebelarse a las regulaciones (Holloway, 2011). A la vez que se opone al concepto de trabajo, el autor precedente refiere a las pequeñas rebeliones a lo que el capital procura imponer. No obstante, más que por las oposiciones de Holloway al concepto de trabajo, aquí sus análisis resultan interesantes por la puesta en relieve de unas potencias creativas que resisten, actúan en las grietas del capitalismo y transforman las regulaciones e imposiciones externas con el hacer cotidiano. Esas potencias interesan porque son expresión de lo que los hombres pueden hacer y, también, de lo que pueden no hacer, es decir, son expresión de "lo que hace que el poder sea poder" (Langer, 2017, p. 61).

Atendiendo a lo anterior, el hacer y el no hacer se vinculan con la potencia de la vida y la producción de lo nuevo (Giorgi y Rodríguez, 2007). Se vuelven creaciones plurales, proponen variaciones al orden establecido (Caruso, 2005). De ahí que esas prácticas no sólo se conciban en términos de obediencia, de consentimiento, sino también de resistencias, conductas y contraconductas. Modos de hacer que -aunque sea parcialmente- se diferencian de y debaten con los modos, modelos y pautas que imperan en la cultura (de Certeau, 2010) escolar. Formas de hacer que, a la vez, interpelan, son parte y dan cuenta de unas políticas de escolarización (Grinberg, 2009) en tanto que producen las condiciones en que se despliega la docencia y disputan los sentidos de las acciones. Las consecuencias y efectos de esos haceres no están definidos de antemano y así debe pensárselos y estudiárselos.

Asimismo, aquí se concibe al trabajo cotidiano de los docentes como prácticas situadas, en tanto que se desarrollan bajo y en función de unas determinadas condiciones sociales, políticas, institucionales pero que en ningún caso constituyen reproducciones lineales de lo establecido explícitamente por las agencias oficiales, aunque sí se vean seriamente afectadas por esas instancias y sus discursividades. Atravesados por las condiciones institucionales que desde esas mismas agencias se generan, los docentes reinterpretan, reformulan, recreándolos, los lineamientos políticos, no sólo los plasmados en los documentos. Es en la conjunción de todas estas condiciones que se produce el hacer docente en las escuelas.

Situar el trabajo cotidiano de los docentes también requiere la atención a las especificidades de ese trabajo, en pos de no perder de vista su "naturaleza propia" (Rockwell, 2018, p. 502). Como expresa la misma autora:

El trabajo docente difícilmente se compara con otros. Su meollo es esa capacidad intangible, intransmisible [...] una habilidad delicada, compuesta de ritmos apropiados, firmeza y confianza mutua en la relación con los alumnos, interés intrínseco de los contenidos y las actividades propuestas, constancia en la conducción, sentido de ritmo y cadencia, entre otros rasgos, que permite lograr convocar a los niños y niñas [o jóvenes] recluidos en el aula a trabajar en lo que se les propone. No es fácil de describir este arte, ni tampoco de transmitirlo [...] (Rockwell, 2018, p. 501).

El debate acerca de si el trabajo de los docentes es un oficio, un arte o una profesión es profuso, de larga data y no está del todo saldado. Aquí se vuelve, si no central, al menos importante darle alguna consideración teniendo en cuenta que la presencia conjunta de esos conceptos -arte, profesión, oficiotensiona el discurso oficial al interpelar a los docentes a asumir una doble posición: trabajadores de la educación y la cultura y profesionales. Actualmente, el concepto de profesionalización está ganando cuerpo en las retóricas oficiales referidas a la docencia y de la mano de lógicas gerenciales. No obstante, expresa la misma autora que, en su especificidad el trabajo de los docentes no puede equipararse ni a las profesiones puesto que no resulta posible la consideración de las escuelas como empresas y los estudiantes y sus familias como clientes, ni a la labor de los artesanos, ni a otros oficios con los que ha querido comparárselos (Rockwell, 2018). De aquí la necesidad de incorporar perspectivas que, junto con caracterizaciones aplicables a la actualidad, permitan comprender a la docencia en la complejidad y en situación. 
Revista de la Escuela de Ciencias de la Educación, año 15, nRo. 14, vol. 2, Julio a Diciembre de 2019. PÁGINAS 51-60. ISSN 18516297 (DESDE DICIEMBRE DE 2006 A DICIEMBRE DE 2017). ISSN 2362-3349 (EN LÍNEA). DOCENCIA, TRABAJO Y DISPUTAS COTIDIANAS EN ESCUELAS SECUNDARIAS DE LAS SOCIEDADES DEL GERENCIAMIENTO. EdUARDO LANGER. GabRIELA ORLANDO.

En cuanto a las formas que asumen el trabajo y el empleo docente en sus aspectos estructurales están profusamente estudiadas (Donaire, 2012; Tenti Fanfani, 2006) para la Argentina actual y en comparación con otros países de América del Sur. Por lo dicho, es que aquí interesa profundizar en cómo se expresa el trabajo específico de los docentes en la imbricación de unas políticas con las vidas laborales y las perspectivas de los propios involucrados. Por una parte, la cuestión del empleo involucra racionalidades y tecnologías de gobierno: los docentes son agentes cuya formación, reclutamiento y desempeño en las escuelas secundarias son regulados; los puestos de trabajo en las escuelas son generados a nivel oficial; el trabajo docente en las escuelas de la Provincia de Buenos Aires, está legal y formalmente especificado. A la vez y, en atención a las racionalidades y puntos de vista de los docentes, el empleo puede aparecer como táctica que atiende tanto a las regulaciones antes referidas como a las propias necesidades, intereses, prospectivas y posibilidades de los sujetos para ser empleados $u$ ocuparse como docentes en las escuelas secundarias. Así, por ejemplo, podrán encontrarse docentes que aprovechan o usan sus saberes y sus titulaciones, se acomodan a los requisitos oficiales para acceder al cargo; hasta se involucran con la docencia -sea iniciando la formación inicial, sea aceptando "reconversiones" propuestas por el Estado, sea decidiendo ejercer con títulos sin orientación pedagógica que los habiliten para serlo- cuando no encuentran espacios laborales en ámbitos extraescolares. Desde ahí, el empleo es también una táctica, un hacer que toma múltiples y particulares formas en la cotidianeidad y que así también merece ser estudiado.

Por último, respecto de la conducción de las conductas, según expresa Dubet (2006) la docencia constituye un "trabajo sobre los otros" (Dubet, 2006, p. 346). No obstante, también es un trabajo hacia los otros, con otros, entre o en medio de otros. Desde la perspectiva de la gubernamentalidad y en sociedades gerenciales, en que las responsabilidades y el eje de las acciones se traslada, como se viene sosteniendo, del Estado y sus grandes instituciones, a los individuos y las comunidades, las prácticas también lo son sobre el self (Grinberg, 2008).

En suma, el trabajo de los docentes no está constituido sólo por sus haceres en las escuelas, aunque éstos constituyan uno de los focos de este artículo. El esfuerzo por asir esa fractalidad para comprenderla, y no para limitarla o fijarla, es el que motoriza los análisis y la escritura de este artículo. Algunos de esos fractales del trabajo y la lucha cotidiana de los docentes en escuelas secundarias del Partido de Gral. San Martín son los que se presentan a continuación. En principio, se refiere a las condiciones en que la autonomía de los docentes se despliega en medio de una serie de condicionantes espaciales. En segundo lugar, se reflexiona sobre los compromisos y las disputas cotidianas que esos mismos docentes desarrollan, en, con y bajo esas condiciones.

\section{"Te acostumbrás a borrar con la mano": regulación y autonomía docente condicionada}

La autonomía se ha vuelto un término muy presente en la retórica de las sociedades de gerenciamiento. Así, en su nombre, en los documentos oficiales de políticas educativas que estructuran la nueva escuela secundaria, se interpela a los sujetos a volverse creativos, flexibles, participativos, comprometidos. Se los invita a hacer autónomamente, a resolver los problemas cotidianos, se los deja hacer, siempre que ese hacer no constituya una disrupción que amenace con el estallido. Los sujetos pueden responder y, de hecho, responden de múltiples maneras a esas interpelaciones: dialogan con ellas, las cuestionan, las aceptan, se adaptan, las consienten, adhieren, las replican y, también, proponen, crean. Sus perspectivas dan cuenta de todas estas cuestiones y se vuelven campo fructífero para el análisis de su trabajo cotidiano y de sus luchas en el entrecruzamiento con las condiciones institucionales presentes.

Se trata de situaciones en que se modifican los términos de gobierno. Son racionalidades que buscan el compromiso y el impulso de los individuos para garantizar la educación de todos y todas (Langer, 2017), delegando, desde ahí gran parte de las responsabilidades en la comunidad educativa. En nuestro trabajo de campo se ha visto cómo, en nombre de esas responsabilidades y compromisos que, en tanto involucran relaciones de poder, son políticos- con las escuelas, con la comunidad y con los jóvenes, los profesores devienen, por ejemplo, recolectores de libros, de fotocopias, ropa para bebés, alimentos o dinero para viajes-; malabaristas y administradores de lo que hay; comunicadores con estudiantes, familias y comunidad toda; transmisores de saberes de múltiples y creativas maneras; sostenedores fervientes de las escuelas en que trabajan y de los procesos de escolarización de los jóvenes. También, cuando las situaciones dejan de ser excepcionales para extenderse a cada vez más cursos, cada vez más días, cada vez más escuelas, es alto el riesgo de agobiarse.

El agobio se presenta cuando los docentes perciben que el Estado no sólo se sirve de ellos una y otra vez, sino que también, actúa reiteradamente con desidia, complicándoles su hacer; condiciona su autonomía, dejando o poniendo en peligro la enseñanza y hasta la vida en las escuelas. Desde ahí, discuten con la retórica oficial de la autonomía. Se trata de discusiones que se juegan, emergen, se expresan en las mismas prácticas discursivas y no discursivas de los docentes en las instituciones. Es a partir de esas discusiones que en las voces de los docentes aparecen, con mucha frecuencia e intensidad, problemas. Las problemáticas atraviesan fuertemente el trabajo cotidiano. Y ello sucede, por ejemplo, cuando las condiciones institucionales llegan a un punto que atentan contra las posibilidades de mantener abierta la escuela. A este respecto, una docente expresa: 
Revista de la Escuela de Ciencias de la Educación, año 15, nRo. 14, vol. 2, Julio a Diciembre de 2019. PÁginas 51-60. ISSN 18516297 (DESDE DICIEMBRE DE 2006 A DICIEMBRE DE 2017). ISSN 2362-3349 (EN LÍNEA). DOCENCIA, TRABAJO Y DISPUTAS COTIDIANAS EN ESCUELAS SECUNDARIAS DE LAS SOCIEDADES DEL GERENCIAMIENTO. EDUARDO LANGER. GabRIELA ORLANDO.

[No hay libros en la escuela] $Y$ los chicos tampoco traen. No, no hay nada. A veces nos cortan la luz. Tuvimos períodos... bueno, a principio de año hubo meses que nos cortaron el agua porque todavía no saben que es una escuela... (Sonrisa que denota entre ironía y resignación) que hay una escuela funcionando ahí (Profesora de Historia, 32 años, octubre 2016).

A la inexistencia de material bibliográfico que obliga a los docentes a ser recolectores de textos, se agregan aquí las cuestiones de infraestructura edilicia, que afectan aún más profundamente, si no cercenan las actividades de enseñanza: los cortes de luz y agua -cuando se producen- vuelven prácticamente imposible hacer algo en esa escuela. Ello en tanto deja a los docentes sin posibilidades de recolectar o reparar ese espacio para que la enseñanza suceda. Si, además, el agua está cortada durante meses enteros, en un lugar donde hay muchas personas, estar en la escuela deviene, cuando menos, inhumano. Se trata de condiciones infraestructurales que, en correlato con las condiciones del barrio, precarizan la vida y el trabajo en las instituciones. Tanto las precarizan que dejan pocas posibilidades de actuar para resolver la cuestión: quedarse a esperar, resignarse a pasar meses enseñando sin agua o con olor a gas, o protestar/ demandar en todas las instancias posibles hasta ser escuchados es algo de lo que se puede hacer y -de hecho- se hace cuando lo que se busca es evitar que se cierre la escuela. Pero la solución queda supeditada a lo que la comunidad educativa o las personas puedan hacer; en el caso antes citado, el agua puede tardar meses en "llegar". Cualquiera de estas opciones afecta considerablemente el trabajo en tanto que posibilidades de enseñar. Aparece una desconsideración de parte de los poderes públicos y de las empresas proveedoras de servicios que se hace más explícita cuando no es que a la escuela nunca llegó el agua: es que la cortan porque "todavía no saben que ahí hay una escuela". Una des-consideración que afecta fuertemente las condiciones de estudio y de trabajo, que es lo que preocupa y entristece a los docentes y que es expresión de precariedad o, con Butler (2010), precaridad. Es decir, ciertas poblaciones que adolecen de redes de apoyo y están diferencialmente expuestas por una condición políticamente inducida en la que "apelan al Estado en busca de protección, pero el Estado es, precisamente, aquello contra lo que necesitan protegerse" (Butler, 2010, p. 46).

La exposición diferencial de ciertas poblaciones a situaciones de precariedad da cuenta de las desigualdades socio-educativas del partido, a la vez que de la desprotección que los docentes perciben de parte del Estado. Esa desprotección de la vida escolar afecta el derecho al trabajo en las condiciones dignas a que aluden los documentos oficiales, ingresa en el discurso político-legal para la educación, pero su efectivización se invisibiliza en la cotidianeidad. Hay casos que resultan todavía más graves y se llevan al extremo como, por ejemplo, el "riesgo de derrumbe". Se reitera en muchos de los entrevistados las advertencias y preocupaciones por los derrumbes de paredes, de techos, de salones. Justamente, el temor a los derrumbes en la escuela es un temor que se funda en experiencias con techos que ya se han caído. Ese temor connota, otra vez, la falta de apoyo que perciben los docentes cuando la vida y el trabajo en la escuela se precarizan. Son situaciones que los mismos docentes definen como nefastas, de descuido de las condiciones de vida y de trabajo por parte de los poderes públicos (Wacquant, 2007) que, además de abonar a las problematizaciones sobre la higiene y seguridad en las escuelas, son reconocidas como mensajes políticos.

Desde ahí, las situaciones descriptas expresan una racionalidad gubernamental que, en esas circunstancias, se vuelve indolente, perezosa, displicente e inerme frente a las necesidades. Lo anterior tiene relación con el papel que estos docentes perciben que juega el Estado. Es un Estado que, más que favorecer el trabajo de los docentes, parece ir, precisamente, en contra de los mismos sujetos a los que llama a hacerse autónomos, responsables de sí mismos, de los otros que forman parte de la vida local y de la escuela. Es un Estado que, como sostiene un profesor "en vez de jugarte a favor, te juega en contra”. Ir contra alguien no es algo que, explícitamente, aparezca en los documentos oficiales. Es algo que se siente, se vive en la cotidianeidad escolar. Un Estado que juega en contra es aquel que mete una escuela en un galpón donde luego vienen a cortar el agua otros empleados públicos que desconocen la existencia de una escuela. Es un Estado que no se fija en qué condiciones se trabaja o estudia y que, cuando reiteradamente se les reclama por reparaciones, "arregla" la acumulación de agua de lluvia en una terraza haciendo, absurdamente, agujeros en los techos de cada salón de clases, tal como sostiene una autoridad escolar:

Como un día estaba inundado, bueno, mandaron gente del Consejo [Escolar] y para sacar el agua del techo, cuando se inunda, hicieron un agujero en el techo de cada aula. En el techo de cada aula. En el techo de cada aula [...] si bien se pusieron unos caños para que después [el agua] saliera al exterior, hicieron un agujero en el techo y un agujero en la pared. En cada aula (enfatiza esta afirmación). Como eso no queda bien sellado y el agua siempre escurre, ahora llueve dentro de las aulas (lo expresa con énfasis). Son cosas que no le caben ni a un chico de primer año. Y no sé si de primer grado, hacer un agujero en el techo. Pero sin embargo acá se hizo. (Vicedirectora, profesora de geografía, 58 años; noviembre 2015).

El descuido de las condiciones de infraestructura llega, en los casos descriptos, hasta lo absurdo. Se trata de situaciones en que los poderes públicos no sólo se muestran como desconsiderados o 
Revista de la Escuela de Ciencias de la Educación, año 15, nRo. 14, vol. 2, Julio a Diciembre de 2019. PÁGinas 51-60. ISSN 18516297 (DESDE DICIEMBRE DE 2006 A DICIEMBRE DE 2017). ISSN 2362-3349 (EN LÍNEA). DOCENCIA, TRABAJO Y DISPUTAS COTIDIANAS EN ESCUELAS SECUNDARIAS DE LAS SOCIEDADES DEL GERENCIAMIENTO. EdUARDO LANGER. GabRIELA ORLANDO.

indolentes, sino también malignamente negligentes (Wacquant, 2007; Burllaile y Orlando, 2009). En esas condiciones trabajan los docentes y son llamados a ser creativos, flexibles, comprometidos y autónomos, por las mismas políticas que los forman como docentes y los vuelve sus empleados. Librados a lo que pueden hacer por sí mismos, aunque reconozcan las contrariedades y contradicciones de la retórica y las prácticas oficiales, sostienen las instituciones, las enseñanzas, los alumnos y, antes que rendirse, aguzan la creatividad y administran las precariedades para evitar cerrar la escuela.

Sin embargo, a pesar de todos sus esfuerzos, las situaciones vinculadas con condiciones de precariedad edilicia configuran el trabajo cotidiano generando vivencias de desborde, de agobio ante las múltiples y recurrentes problemáticas que a diario atraviesan las instituciones. Y, por más comprometidos y responsables que se sientan y que sean, el sentirse excedido en las posibilidades de acción para resolver es la constante. $Y$ cuando no hay para resolver, cuando la precariedad para administrar es demasiado grande, hasta "te acostumbrás a borrar con la mano" (Jefa de área y profesora de Geografía y Ciencias Sociales, 34 años; Octubre 2016).

En estas condiciones de fragilidad que es mucho más que edilicia, sostener la escuela se vuelve una tarea compleja que tensiona el deseo y las voluntades con las frustraciones y el cansancio ya que "no hay voluntarismo que pueda resolver la precaridad forzosa que caracteriza la biopolítica contemporánea” (Grinberg y Bussi; 2015, p. 12). Acostumbrarse, pasa, así, a formar parte de las actividades de los docentes. Junto con los deseos y las preocupaciones, el acostumbrarse a que no hay borrador, a que hay que abrir la escuela aunque le corten durante meses el agua, forma parte de esa autonomía a la vez condicionada y lesionada. Aun con todas esas vicisitudes, estos docentes no se amedrentan. En lugar de aceptaciones sumisas, producen respuestas cotidianas a través de sus formas de comprometerse y de participar para sostener la escuela y disputar para que mejoren las instituciones y los procesos de enseñanza y aprendizaje. Es a esos compromisos y disputas en la cotidianeidad, tal como las relatan los docentes que trabajan en escuelas secundarias del partido de Gral. San Martín, que se refiere a continuación.

\section{Compromisos y disputas cotidianas}

En los trabajos de extensión e investigación nos encontramos con docentes que además de la denuncia por las condiciones que denotan la precariedad y la degradación, producen respuestas que están cargadas de deseos de una escuela mejor, tal como la docente que sigue expresa en torno a una escuela acogedora en la que todos los actores estén comprometidos:

Quiero imaginarme una escuela más poblada [...] Quiero ver docentes comprometidos, que vayan porque aman [...] Espero ver padres más comprometidos en esa escuela [...] es muy importante que tu hijo te vea siendo parte de la escuela (Jefa de área y profesora de Geografía y Ciencias Sociales, 34 años; Octubre 2016).

Aparecen, en esta última frase, los "quiero" y los "espero" en torno a la cuestión de los compromisos. Sostenemos que son deseos que conllevan disputas o, como dice Deleuze (1995), que llevan las líneas de fuga mediante afectividades que expresan frente a sus estudiantes y ante condiciones determinadas. En esos deseos y linealidades entre compromiso, participación y autonomía es que se encuentran las luchas, los debates y las disputas que forman parte de los discursos que se presentan en la voz de los docentes entrevistados. Contra los discursos que acusan a los docentes de estar descomprometidos, que no trabajan, que se ausentan, éstos demandan a los demás y a sí mismos, como colectivo, el comprometerse (Langer, 2017). Comprometerse es trabajar y muchas otras cosas más que hacen al entendimiento, a la comprensión de las situaciones, al acompañamiento, la contención y, por sobre todo, la presencia. Es "estar arriba de los chicos" ante situaciones que desbordan todo el tiempo en las escuelas en contextos de pobreza urbana, tal como señala aquí una de las profesoras entrevistadas:

Uno no sabe cómo ayudarlos [...] por suerte, gran parte de los profes saben dónde están trabajando, son muy comprometidos. Incluso, te cuento dos segundos una situación con una alumna... (Se le llenan de lágrimas los ojos), ay perdón (se larga a llorar). G... (nombre de la alumna), está sola. Vive en un galpón con la tía. Y la tía no tiene para darle de comer. El colegio le da la comida y [dice todo esto llorando] una profe se hace cargo de ella, de comprarle los útiles y demás. Ehhhh. (no puede seguir hablando). Los profes están muy arriba de los chicos. $Y$ se hace lo que se puede. [...] Sabe que la queremos ayudar. Estamos ahí para ayudarla. Como ella hay treinta más. Que son situaciones que desbordan. Pero por suerte los profes charlan mucho con los chicos. Y bueno al menos esas seis horas que están en el colegio sabemos que están bien" (profesora de Historia, 32 años; Octubre, 2017).

En este enunciado se remarca el querer ayudar a los estudiantes y no poder, lo que constituye otra afección, otro condicionamiento a la autonomía. Aparecen, una vez más, referencias a situaciones que desbordan y el estar solo para ayudar. Hay una estudiante sola, con una tía sola, que es ayudada por docentes que también están solos para atender esa situación, que de por sí es desbordante y que 
Revista de la Escuela de Ciencias de la Educación, año 15, nRo. 14, vol. 2, Julio a Diciembre de 2019. PÁGINAS 51-60. ISSN 18516297 (DESDE DICIEMBRE DE 2006 A DICIEMBRE DE 2017). ISSN 2362-3349 (EN LÍNEA). DOCENCIA, TRABAJO Y DISPUTAS COTIDIANAS EN ESCUELAS SECUNDARIAS DE LAS SOCIEDADES DEL GERENCIAMIENTO. EDUARDO LANGER. GabRIELA ORLANDO.

se multiplica en otras treinta soledades que en la escuela hay que atender. No hay nadie que ayude, más que los propios docentes. Son esos otros con quienes comparten una institución y unas vidas que han sido arrojados a su suerte, con los que se cuenta. Y, sin embargo, en esas soledades multiplicadas, se hace lo que se puede frente a situaciones cuya resolución o atenuación resulta imposible. Y, no obstante, es en la escuela donde se está bien, porque afuera de la escuela se está mal, o se está peor. Ante ese cúmulo de situaciones desbordantes, cuando no caen en la impotencia, los docentes viven tensiones y contradicciones por encontrar sentido a lo que hacen y poder enseñar. Porque en su materia se esfuerzan por generar espacios de enseñanza, de transmisión de saberes escolares, "dan los aztecas", tal como refería esa misma docente y, mientras tanto, saben que nadie, salvo ellos mismos, se ocupa de los cuidados más básicos de las vidas de los estudiantes: "Alguien se debería hacer cargo, y nadie se hace cargo. $Y$ uno trabaja como puede. Y llegas al punto de decir, ¿qué les sirve a estos chicos? No sé" (Profesora de Historia, 32 años; Octubre 2017). Pedir que otros, u otros también, u otros junto con ellos se hagan cargo y seguir trabajando, preguntarse por los sentidos, estar y ser desbordados por las situaciones son todos condimentos que hacen a estar comprometido e ir mucho más allá de lo que establece y requiere cualquier normativa y política en términos laborales. Llorar y sufrir en carne propia esos efectos, tal como lo hace esta docente, implica mucho más que ir, estar y enseñar en la escuela. Se trata de sujetos para los que el compromiso significa que docentes, estudiantes, familias, estén presentes, pero no de cualquier manera. Que vayan a la escuela porque aman y porque es importantísimo que los jóvenes vean que "sos parte"; seas profesor, familia o compañero, lo central es participar. Quieren y desean una escuela comprometida y ello a partir de pensar a los docentes, a los directivos, al Estado y al acompañamiento familiar de manera comprometida. Entonces, el compromiso remite a las presencias y a las participaciones en la cotidianeidad, y eso no es casual tratándose, como se dijo, de docentes que las ejercen, las valoran y las exigen. Así lo expresa la siguiente docente respecto del futuro que imagina para una escuela que se está transformando: "Me veo participando. Me gustaría, me gustaría ser parte. Porque si no, es como... estar luchando contra la corriente. No quisiera. No quisiera estar renegando los últimos años como docente (Ríe)" (Profesora de Historia y Jefa de Área de Ciencias Sociales, 34 años; Octubre 2016).

Nuevamente, a sus dudas respecto de las retóricas de una nueva escuela se suceden los deseos en las disputas cotidianas. Sin ellos, los docentes no podrían continuar, insistir, enseñar. Por tanto, participar, desde este punto de vista, es luchar, ir con la corriente y no en su contra, porque son docentes que tienen esperanzas, quieren que sus estudiantes luchen, intentan que ellos también puedan tener esperanza y que defiendan sus derechos, disputan para que la educación "no decaiga" y se transforme en función de los sentidos que le otorgan las nuevas generaciones.

En estas tensiones entre el agotamiento, el tener que hacer todo solo, el querer pero no poder, la depresión y angustia que generan las situaciones y el entusiasmo, el deseo, los intentos, la onda y las ganas describimos esas disputas cotidianas en las escuelas. Por ello, ser comprometido y participar, también, para los docentes es tratar de pensar y hacer algo para que la educación pueda ser transformada y que, en ese proceso, los diferentes actores de la comunidad tengan la posibilidad de estar activamente.

Esas disputas cotidianas ocurren y se vislumbran como posibilidad de pensar y hacer una mejor escuela todos los días con, como decía un docente, la "participación real" de diferentes actores tales como los docentes, los sindicatos, los políticos, los padres, la universidad, etc. A la vez, con el sueño de que en algún momento esa educación se transforme, para "solucionar lo obsoleto". Si bien parecerían ser críticas radicales a todo el proceso educativo, desde los discursos docentes aquí analizados, se piensa y problematiza aquello que sucede todos los días en las aulas de las escuelas: el hacer todo al mismo tiempo expresa, quizás, el gran problema cotidiano que existe en estas escuelas.

\section{Comentarios finales}

El trabajo de los docentes está constituido por entrecruzamientos de prácticas en una complejidad de condiciones sociales, institucionales y múltiples agenciamientos -colectivos y singularesEn este sentido, en la cotidianeidad de las escuelas secundarias, de todas y cada una, el trabajo de los docentes ocurre como una particular combinación de políticas de escolarización que involucran condiciones, capacidades, saberes, racionalidades, tácticas y estrategias que expresan modos de actuar de los sujetos sobre y consigo mismos a la vez que entre, sobre y con otros.

Esas expresiones muestran consentimientos, aceptaciones y también disputas; proponen nuevos órdenes, transforman. Son creaciones múltiples, complejas, fractales que, como los copos de nieve o las huellas digitales, asumen formas singulares y colectivas a la vez, se combinan, comunican, comparten rasgos estructurales sin que haya uno idéntico a otro. Desde aquí, el centro estuvo en describir el trabajo cotidiano de los docentes en el marco de las regulaciones actuales y los condicionamientos a la autonomía y luego, sus disputas cotidianas en las escuelas.

En relación con lo primero, aparecen en los entrevistados, enunciados que denotan percepciones de impotencia ante situaciones que son casi imposibles de resolver en y con los modos y recursos en que el discurso oficial apela a resolverlas; la sensación de que están solos y sus reclamos no son oídos; el tiempo que pasa hasta que, ante tantas demandas, alguien llega a la escuela y, cuando llega, puede ser que, en lugar de arreglar la infraestructura, destruya lo sano; la desconfianza hacia unas políticas que los deja librados a hacer como pueden y con lo que tienen en nombre de una autonomía 
Revista de la Escuela de Ciencias de la Educación, año 15, nRo. 14, vol. 2, Julio a Diciembre de 2019. PÁginas 51-60. ISSN 18516297 (DESDE DICIEMBRE DE 2006 A DICIEMBRE DE 2017). ISSN 2362-3349 (EN LÍNEA). DOCENCIA, TRABAJO Y DISPUTAS COTIDIANAS EN ESCUELAS SECUNDARIAS DE LAS SOCIEDADES DEL GERENCIAMIENTO. EdUARDO LANGER. GabRIELA ORLANDO.

lesionada. Los docentes enuncian y dan cuenta de la exposición diferencial a situaciones de precariedad, las desconsideraciones, las desprotecciones y/o la falta de apoyo por parte del Estado, que no juega a favor sino en contra de las instituciones y de los sujetos que allí están cotidianamente presentes.

En segundo lugar, en relación con las disputas, se describieron sus compromisos, sus participaciones, sus múltiples intentos, las insistencias, el no bajar los brazos a pesar de todo. Son docentes que, entienden, no pueden solucionar los problemas estructurales que tienen sus estudiantes "soluciones concretas no les podemos dar"-, porque, muchas veces, en contextos de extrema pobreza y desigualdad urbana, ni ellos mismos ni las escuelas, cuentan, en la soledad con herramientas o con recursos suficientes para afrontar tanta precariedad. $Y$ decimos muchas veces porque, efectivamente, esas veces son muchas.

En definitiva, son docentes que, además de enseñar a los jóvenes lo que corresponde a sus procesos de escolarización, lo pautado y lo esperable de ser enseñado en una escuela secundaria, los escuchan, los aconsejan, los contienen todos y cada uno de los días, se preguntan por los sentidos de la educación en estas realidades, piensan qué hacer mejor y de qué formas. En ese contexto, transmiten saberes, se ven afectados, lloran y sufren por las situaciones que viven sus estudiantes. En esas condiciones, esto constituye un llamado a luchar porque, tal como decía un docente, "cuando creas un buen vínculo con un grupo, cuando se comprometen con algo, eso es, yo, lo que más lucho". El compromiso, su compromiso, deviene en el estar, el acompañar, el sostener y el desear, con esos otros arrojados a la intemperie, una escuela mejor.

\section{Notas Bibliográficas}

(1) Se hace referencia a las recientes lamentables muertes de dos personas en una escuela en Moreno que, en parte, también, motivan esta escritura.

(2) En el marco del CEDESI (Centro de Estudios sobre Desigualdad, Sujetos e Instituciones) de la Universidad Nacional de San Martín, Provincia de Buenos Aires, Argentina se realizaron entrevistas a veinte docentes de 23 escuelas secundarias según criterios de georreferencia y en función de un índice de vulnerabilidad socio geográfico de las escuelas.

(3) El taller "es un espacio que tiene como objetivo la producción de audiovisuales por parte de estudiantes con el fin de propiciar situaciones de pensamiento y problematización de la realidad a través de la realización documental, a la vez que generar debates e intercambios de las problemáticas barriales en el espacio escolar" (Carpentieri, Dafunchio, Langer y Machado, 2015: 1). El taller se realiza desde 2008 a la actualidad, una vez por semana, con estudiantes del nivel secundario de entre 12 y 19 años de una escuela que se sitúa a pocas cuadras de dos villas miseria del Área Metropolitana de Buenos Aires y que en cada año se finaliza con la producción de cortos documentales. Aquí se traen a colación algunos breves retazos de alguno de ellos.

(4) Se refiere a la Feria de Ciencias Humanas y Sociales y al Festival de Cortos Audiovisuales para todas las escuelas secundarias del Partido de San Martín organizada por la Universidad Nacional de San Martín, coordinadas por el Dr. Eduardo Langer. Para más información ver http://noticias.unsam.edu.ar/2018/4/11/30feria-de-cs-humanas-y-sociales/

\section{Referencias Bibliográficas}

- Beltrán Llavador, F. (Coord.). (2006). La gestión escolar de los cambios del currículum en la enseñanza secundaria. Buenos Aires: Miño y Dávila.

- Butler, J. (2010). Marcos de guerra. Las vidas lloradas. Buenos Aires: Paidós.

- $\quad$ Carpentieri, Y.; Dafunchio, S.; Langer, E. y Machado, M. (2015). Producir saberes desde la experiencia de un taller audiovisual en una escuela secundaria en contextos de pobreza urbana. Novedades Educativas (294), pp. 27-32.

- Caruso, M. (2005). La biopolítica en las aulas. Buenos Aires: Prometeo.

- De Certeau, M. (2010). La invención de lo cotidiano. México: Universidad Iberoamericana.

- Deleuze, G. (1995). Deseo y placer. Archipiélago: cuadernos de crítica de la cultura, (23), pp. 12-20.

- Deleuze, G. (2015). La subjetivación. Buenos Aires: Cactus.

- Donaire, R. (2012). Los docentes en el siglo XXI: ¿empobrecidos o proletarizados? Burzaco. Argentina: Siglo XXI.

- Dubet, F. (2006). El declive de la institución. Profesiones, sujetos e individuos en la modernidad. Barcelona: Gedisa.

- Ezpeleta, J. (1991). Escuelas y maestros. Condiciones del trabajo docente en la Argentina. Buenos Aires: Centro Editor de América Latina.

- Feldfeber, M. (2007). La regulación de la formación y el trabajo docente: un análisis crítico de la "agenda educativa" en América Latina. Educação e Sociedade, (28)99. 444-465. Recuperado de: http://www.cedes.unicamp.br

- Foucault, M. (2002). Vigilar y castigar. Nacimiento de la prisión. Buenos Aires: Siglo XXI.

- Foucault, M. (2006). Seguridad, territorio, población: curso en el Collége de France: 1977-1978. Buenos Aires: Fondo de Cultura Económica.

- Foucault, M. (2007). Nacimiento de la biopolítica. Curso en el Collège de France (1978-1979). Buenos Aires: Fondo de Cultura Económica.

- Foucault, M. (2007a). La gubernamentalidad. En GIORGI, G. y RODRíGUEZ, F. (Comps.). Ensayos sobre biopolítica. Excesos de vida, pp. 187-215. Buenos Aires: Paidós. 
Revista de la Escuela de Ciencias de la Educación, año 15, nRo. 14, vol. 2, Julio a Diciembre de 2019. PÁginas 51-60. ISSN 18516297 (DESDE DICIEMBRE DE 2006 A DICIEMBRE DE 2017). ISSN 2362-3349 (EN LÍNEA). DOCENCIA, TRABAJO Y DISPUTAS COTIDIANAS EN ESCUELAS SECUNDARIAS DE LAS SOCIEDADES DEL GERENCIAMIENTO. EDUARDO LANGER. GABRIELA ORLANDO.

- Foucault, M. (2009). El gobierno de sí y de los otros. Buenos Aires: Fondo de Cultura Económica.

- Giard, L. (2010). Presentación. Historia de una investigación. En De Certeau, M. La invención de lo cotidiano. I. Artes de hacer. México: Universidad Iberoamericana.

- $\quad$ Giorgi, G. y Rodríguez, F. (Comps.). (2007). Ensayos sobre biopolítica. Excesos de vida (pp. 187-215). Buenos Aires: Paidó,.

- Grinberg, S. (2008). Educación y poder en el siglo XXI. Gubernamentalidad y pedagogía en las sociedades de gerenciamiento. Buenos Aires: Miño y Dávila.

- Grinberg, S. (2009). Políticas y territorios de escolarización en contextos de extrema pobreza urbana Dispositivos pedagógicos entre el gerenciamiento y la abyección. Archivos de Ciencias de la Educación, 3(3), pp. 81-98.

- $\quad$ Grinberg, S. y Bussi, E. (2015). La escuela en la encrucijada. Superdocentes y precariedad/precaridad cotidiana en contextos de extrema pobreza urbana y degradación ambiental. En Prensa: Mimeo.

- Holloway, J. (2011). Agrietar el capitalismo. El hacer contra el trabajo. Buenos Aires: Herramienta.

- Imen, P. (2008). Trabajo docente: debates sobre autonomía laboral y democratización de la cultura. En FELDFEBER, M. y ANDRADE OLIVEIRA, D. (Comps.) Políticas educativas y trabajo docente. Nuevas regulaciones. ¿Nuevos sujetos?, pp. 239-261, Buenos Aires: Noveduc.

- Langer, E. (2016). La noción de «contraconductas» de Foucault y su centralidad para caracterizar los procesos de escolarización en el siglo XXI. En Langer, E. y Buenaventura Rodríguez, B. (Comps.). Usos y prospectivas de Foucault en la educación a 30 años de su muerte, pp. 265-283. UNSAM-UNPA. Argentina: Rada Tilly, Del gato gris.

- $\quad$ Langer, E. (2017). Escuela, pobreza y resistencia. Defensas y luchas cotidianas de estudiantes. Argentina: Rada Tilly, Del gato gris.

- Lazzarato, M. (2010). Políticas del acontecimiento. Buenos Aires: Tinta Limón.

- Miller, P. y Rose, N. (1990). Governing economic life. Economy and Society, 19(1), pp. 1-31.

- Murúa, O.; Orlando, G. y Paredes, L. (2016). Von Abwesenden mit Anweseneit und Anwesenden mit Abwesenheit. Unregelmäßige Schulbesuche und neue Modi des Schülerdaseins in der Sekundarstufe. En: CLEMENT, U. y OELSNER, V. (Eds.). Was match schule? Schule als gestalteter Raum, pp. 179-190, Springer, Germany.

- O’Malley, P. (2014). Riesgo, neoliberalismo y justicia penal. En Rodríguez Freire, R. Evaluación, gestión y riesgo. Para una crítica del gobierno del presente, pp. 103-140. Santiago de Chile: Universidad Central de Chile.

- Rockwell, E. (2018). Vivir entre escuelas. Relatos y presencias. Antología esencial. CLACSO. Recuperado de: http://biblioteca.clacso.edu.ar/clacso/se/20180223024326/Antologia_Elsie_Rockwell.pdf

- Rose, N. (2007). ¿La muerte de lo social? Re-configuración del territorio de gobierno. Revista argentina de sociología, 5(8), pp. 111-150.

- Tenti Fanfani, E. (Comp.). (2006). El oficio de docente. Vocación, trabajo y profesión en el siglo XXI. Buenos Aires: Siglo XXI.

- Wacquant, L. (2007). Los condenados de la ciudad. Gueto, periferias y Estado. Buenos Aires: Siglo XXI. 